\title{
Protocol
}

\section{In Vivo Detection of Fluctuating Brain Steroid Levels in Zebra Finches}

\author{
Maaya Ikeda, ${ }^{1,3}$ Michelle A. Rensel, ${ }^{2}$ Barney A. Schlinger, ${ }^{2}$ and Luke Remage-Healey ${ }^{1,3,4}$ \\ ${ }^{1}$ Neuroscience and Behavior Program, Center for Neuroendocrine Studies, University of Massachusetts, Amherst, \\ Massachusetts 01003; ${ }^{2}$ Department of Integrative Biology and Physiology, University of California, Los Angeles, \\ Los Angeles, California 90095; ${ }^{3}$ Molecular and Cellular Biology Program, University of Massachusetts, Amherst, \\ Massachusetts 01003
}

This protocol describes a method for the in vivo measurement of steroid hormones in brain circuits of the zebra finch. A guide cannula is surgically implanted into the skull, microdilysate is collected through a microdialysis probe that is inserted into the cannula, and steroid concentrations in the microdialysate are determined using the enzyme-linked immunosorbent assay (ELISA). In some cases, the steroids measured are derived locally (e.g., neural estrogens in males), whereas in other cases, the steroids measured reflect systemic circulating levels and/or central conversion (e.g., the primary androgen testosterone and the primary glucocorticoid corticosterone). A reverse-microdialysis ("retrodialysis") method that can be used to deliver pharmacological agents into the brain to influence local steroid neurochemistry as well as behavior is also discussed.

MATERIALS

It is essential that you consult the appropriate Material Safety Data Sheets and your institution's Environmental Health and Safety Office for proper handling of equipment and hazardous material used in this protocol.

RECIPE: Please see the end of this protocol for recipes indicated by $<R>$. Additional recipes can be found online at http://cshprotocols.cshlp.org/site/recipes.

\section{Reagents}

Artificial cerebrospinal fluid (aCSF) for zebra finch brain $<\mathrm{R}>$

The bovine serum albumin (BSA) in the aCSF increases the solubility of lipophilic steroids (Remage-Healey et al. 2008, 2010); however, BSA causes interference with the ELISA for corticosterone. When measuring brain corticosterone levels, use BSA-free Dulbecco's phosphate-buffered saline (Sigma-Aldrich) instead of aCSF.

ELISA kit to detect the steroid of interest Corticosterone EIA kit (Cayman Chemical; cat. no. 500655) $\mathrm{E}_{2}$ EIA kit (Cayman Chemical; cat. no. 582251) for 17- $\beta$-estradiol Testosterone ELISA kit (Enzo Life Sciences; cat. no. ADI-900-065)

Equithesin

We use an in-house formulation of this general anesthetic that contains $10.2 \mathrm{mg} / \mathrm{mL} \mathrm{Nembutal,} 42.5 \mathrm{mg} / \mathrm{mL}$ chloral hydrate, $34.6 \%$ propylene glycol, $8.9 \%$ ethanol, and $21 \mathrm{mg} / \mathrm{mL}$ magnesium sulfate. Equithesin is administered intramuscularly at a dose of $3.2 \mathrm{mg} / \mathrm{kg}$ body weight. Isofluorane can be used as an alternative.

\footnotetext{
${ }^{3}$ Correspondence: healey@cns.umass.edu

(C) 2014 Cold Spring Harbor Laboratory Press

Cite this protocol as Cold Spring Harb Protoc; doi:10.1101/pdb.prot084616
} 
M. Ikeda et al.

Ethanol (20\% and 100\%)

Lidocaine (2\% in ethanol) or another local anesthetic

Meloxicam

Zebra finches (males and females; age $>120 \mathrm{~d}$ )

All finches used in our experiments are from our institutional breeding colony.

Equipment

Acoustic attenuation chamber (Audiometric Booth AB08; Eckel Industries)

The chamber has a one-way glass partition and a 10-mm portal for microdialysis inflow/outflow tubing.

Cotton-tipped applicators

Cyanoacrylate adhesive (e.g., Vetbond)

Dental acrylic (Perm Reline and Repair Resin; Hygenic; cat. no. H00358)

Dissection microscope (Zeiss OPMI 1)

Fluorinated ethylene propylene (FEP) tubing and adaptors (CMA Microdialysis)

Fraction collector (refrigerated) (CMA 470) (CMA Microdialysis)

This is optional, but it is very useful for the collection of large numbers of samples.

Guide cannula (CMA 7) (CMA Microdialysis)

Heating pad (FHC)

Kimwipes

Microdialysis cage

Microdialysis probe (CMA 7; $1 \mathrm{~mm}$ cuprophane probe membrane) (CMA Microdialysis)

The probe membrane has a 6-kDa cutoff, which allows the passive diffusion of small molecules and pharmacological agents such as steroids and enzyme inhibitors ( $300 \mathrm{Da})$.

Microdialysis tubing adaptors (for connection to swivel) (Instech MC015)

Needles (22-, 26-, and 30-gauge)

Probe/guide clip (CMA)

Standard surgical tools

Stereotaxic apparatus (adapted for use with small birds)

We use a device that was custom-made by Herb Adams Engineering.

Swivel (dual-channel, quartz-lined) (Instech 375/D/22QM)

Syringe, for injections $(0.5-\mathrm{cc})$

Syringe, for pump (1.0-cc; set to infuse at $2.0 \mu \mathrm{L} / \mathrm{min})$

Syringe filters $(0.22-\mu \mathrm{m})$

Syringe pump (PHD 22/2000 Infusion from Harvard Apparatus)

Tether (a modified sleeve that connects the swivel to the cannula; see Fig. 1)

A counter-balanced lever arm can be obtained from Instech for microdialysis studies with mice.

\section{METHOD}

Surgery

The surgical procedure takes $30 \mathrm{~min}-1 \mathrm{~h}$.

1. Weigh the zebra finch. Deprive the animal of food for $\sim 40 \mathrm{~min}$ to allow the crop to empty and to prevent aspiration pneumonia during surgery.

2. Incubate the Equithesin in a warm water bath to dissolve any crystals in the solution.

3. Administer an intramuscular injection of $50 \mu \mathrm{L}$ of Equithesin to the breast muscle of the finch. For animals $<100 \mathrm{~d}$ posthatch or $<12 \mathrm{~g}$ in weight, inject 30-45 $\mu \mathrm{L}$ of Equithesin.

For Equithesin-induced anesthesia, breathing rates should be short and shallow. Wait 20 min until the bird is deeply anesthetized. If breathing is labored, terminate surgery immediately. 


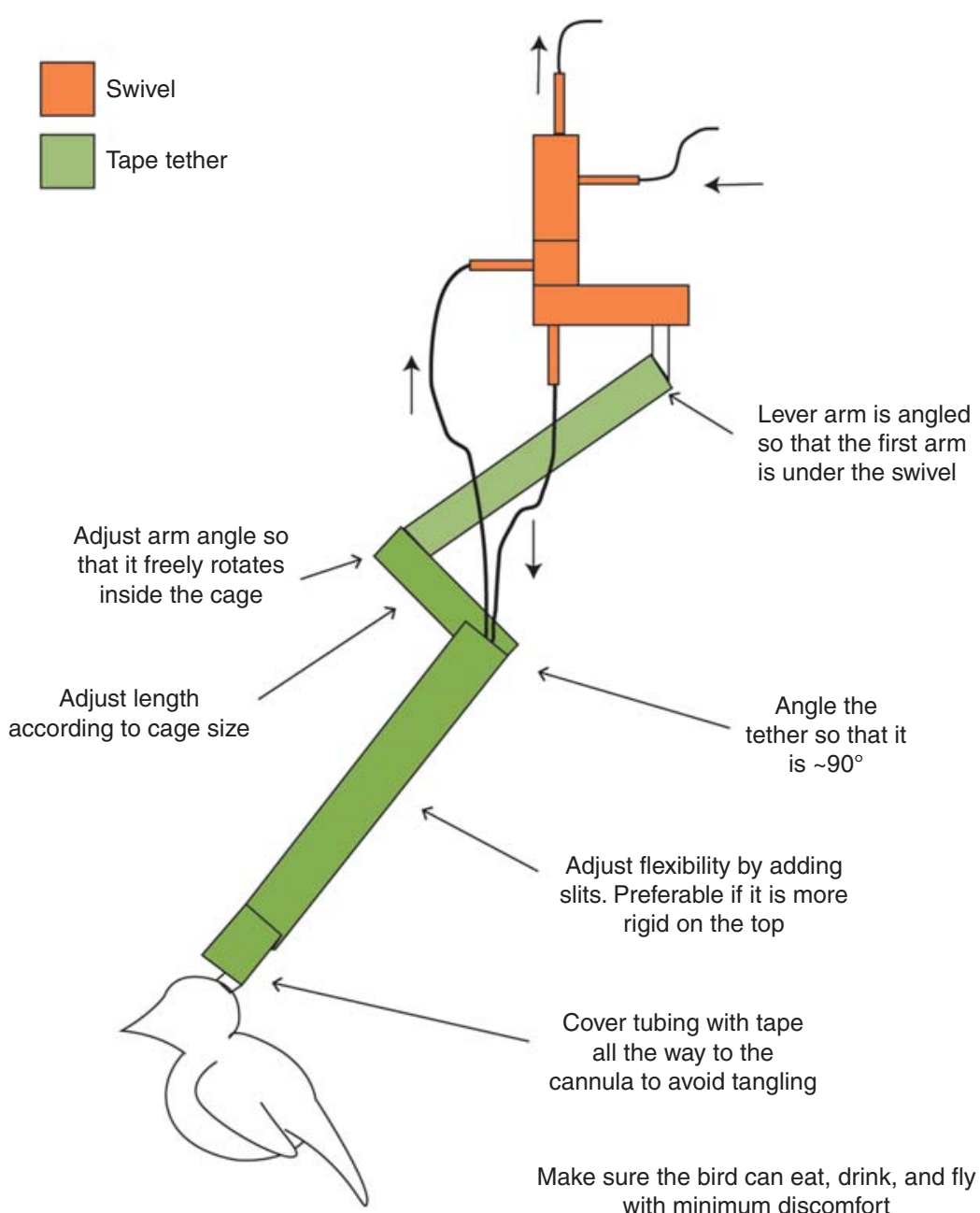

FIGURE 1. Schematic representation of the customizable tether design used in zebra finch in vivo microdialysis. At the top is the swivel connected to the microdialysis cage with inlet and outlet FEP tubing. The top of the tether (lever arm) contains either a thin wire or plastic straw (such as those that are shipped with CMA 7 probes to protect them) to enable lightweight rigidity, and it is then enshrouded with tape. The tether is angled to provide a lever arm to translate rotational torque from the bird to the swivel while also allowing full freedom of movement (including flight) inside the cage. Multiple tape component stages ensure flexibility in addition to an angled lever arm.

For isoflurane-induced anesthesia, use $2 \%-3 \%$ isoflurane with $0.4 \mathrm{~L} / \mathrm{min}$ oxygen. Monitor the depth of anesthesia as described above; isoflurane typically takes effect within $5 \mathrm{~min}$. Incrementally decrease the isoflurane throughout the procedure to prevent overdose while maintaining the depth of anesthesia.

4. Wrap the bird in a Kimwipe "tunic" and bind the body loosely with tape.

Animals are more comfortable during survival surgery if they are lightly restrained (Grandin 2007).

5. Remove the feathers from the head and around the ear canal using scissors. Alternatively, brush the head feathers back gently with a cotton-tipped applicator soaked in $20 \%$ ethanol.

6. Secure the bird on a stereotaxic apparatus on top of a heating pad set at $\sim 34^{\circ} \mathrm{C}$.

Set the angle of the head to ensure the skull plane of the target area is perpendicular to the cannula entry point. For implantations targeting the caudal telencephalon, this head angle is typically $45^{\circ}$.

7. Inject $10-15 \mu \mathrm{L}$ of $2 \%$ lidocaine subcutaneously into the scalp.

Smooth the lidocaine into the working area and confirm that the local anesthetic is effective by testing the scalp with light forceps.

8. Using iridectomy scissors, make incisions to the skin along the midline and expose the skull. Make lateral incisions from this medial incision at both rostral and caudal ends, forming an "I." 
M. Ikeda et al.

9. Locate the bifurcation of the midsagittal sinus (point-of-origin).

This can be best accomplished by making a small window incision (remove a $1 \mathrm{~mm} \times 1 \mathrm{~mm}$ ) in the upper leaflet of skull surrounding the sinus area. Leave the lower leaflet intact.

10. Secure a 26-gauge needle to a probe/guide clip attached to a micromanipulator and place the tip of the needle on top of the point-of-origin ("zero point") without piercing the lower leaflet. Note the coordinates on the micrometer scales for $x$ and $y$ dimensions on the stereotaxic apparatus.

11. Using stereotaxic coordinates create a small hole through both upper and lower leaflets of the skull using needle tips.

12. Carefully resect the dura mater inside the hole using a 30-gauge needle and expose the brain surface.

A shallow lateral resection is essential to avoid damaging underlying brain tissue.

13. Descend the guide cannula ventral to the target area using preestablished depth coordinates. Leave the obdurator (the "dummy probe" supplied with the CMA cannula) inside the guide cannula.

14. Secure the guide cannula to the skull by applying a thin layer of cyanoacrylate adhesive around the opening, avoiding direct contact with the surface of the brain.

For subadults, use a \#11 scalpel blade to etch small, shallow lines in the skull area roughening the skull surface and encouraging cyanoacrylate adhesion.

15. While the cyanoacrylate is drying, apply dental acrylic on top of it using cotton swabs. Layer additional cyanoacrylate as necessary.

Make sure that the dental acrylic completely covers the guide shaft of the cannula and at least one third of the cannula housing itself.

16. Reattach the scalp to all remaining exposed skull areas by applying cyanoacrylate under the skin and securing it to the skull and dental acrylic.

17. After recovery, monitor and treat the animal for perioperative pain as necessary with meloxicam (or another nonsteroidal anti-inflammatory drug).

Probe Implantation

Perform probe implantation 3-4 d after surgery. The procedure takes $\sim 1 \mathrm{~h}$.

18. Fill FEP tubing and the swivel assembled to the microdialysis cage with aCSF.

19. Prime the microdialysis probe by placing it in the probe clip and immersing it in $100 \%$ ethanol in a microcentrifuge tube. Keeping the probe immersed, pump water (for $20 \mathrm{~min}$ ) and then aCSF ( $8 \mu \mathrm{L} / \mathrm{min}$ ) into the probe through the inlet tube and attach a microdialysis tether to the probe using a small piece of tape. After flushing, remove the probe from the microcentrifuge tube and implant it in the guide cannula (see Step 20).

This transfer should be completed quickly ( $<1 \mathrm{~min})$ so that the microdialysis probe does not dry out.

20. Grasp the bird in one hand, stabilize the head gently with fingertips and, using \#5 forceps, remove the dummy probe from the guide cannula and replace it with the prefilled microdialysis probe.

Implantation can be completed under light isoflurane anesthesia to minimize disturbance.

21. Apply small amount of cyanoacrylate adhesive to the exterior of the probe housing at the top of the cannula to keep the probe in place.

Make sure the cyanoacrylate is external to the cannula channel to not impact the CNS or probe, and doublecheck that aCSF is welling out from the outlet tubing after the implantation.

22. Connect the inlet and outlet tubing to the microdialyis tubing inside the chamber.

23. Attach the tether to the arm as shown in Figure 1. Adjust the tether height and lever arm, and confirm that dialysate is flowing at the correct rate.

Most ELISAs require at least $50 \mu \mathrm{L}$ of sample. A flow rate of $2 \mu \mathrm{L} / \mathrm{min}$ for $30 \mathrm{~min}$ is sufficient to obtain such samples. See Troubleshooting. 
Detecting Brain Steroid Levels in Zebra Finches

\section{Detection of Steroids}

24. Begin in vivo experiments $8-12 \mathrm{~h}$ following implantation to allow implantation-induced neurochemical responses to subside. Use a commercially available ELISA kit to detect steroids in the dialysate.

In most cases, the dialysate can be run directly on the ELISA plate because our aCSF with BSA is similar to the composition of the ELISA assay buffer. Always run four to eight control wells for each ELISA with aCSF that has not been perfused through the dialysate system to establish baseline comparisons.

\section{TROUBLESHOOTING}

Problem (Step 23): The aCSF dialysate is not flowing at the correct rate.

Solution: When the bird is removed from the system, flush $\mathrm{ddH}_{2} \mathrm{O}$ through the entire system (FEP tubing, swivel inlet, and outlet) for $60 \mathrm{~min}$ after every use at $8-10 \mu \mathrm{L} / \mathrm{min}$. Continue flushing $\mathrm{ddH}_{2} \mathrm{O}$ at $0.5-1.0 \mu \mathrm{L} / \mathrm{min}$ until the next experiment. Filter the aCSF perfusate through a $0.22-\mu \mathrm{m}$ syringe filter before pumping it through the microdialysis setup to prevent particulate buildup.

Problem (Step 23): The FEP tubing is clogged (i.e., there is no flowthrough a section of the dialysis setup).

Solution: Double-check that fluid is welling from the syringe tip at the desired flow rate. Run the system at $8 \mu \mathrm{L} / \mathrm{min}$ for $\sim 1 \mathrm{~min}$ to try to flush bubbles or particulate matter from the tubing. Check that fluid is coming out of each connection of FEP tubing. Trim the ends of the FEP tubing at each connection to remove clogs.

Problem (Step 23): The clog is isolated to the bird (within the probe tubing).

Solution: Make sure that the FEP tubing in the tether is not tangled. Remove the tape around the FEP tubing and reapply to reduce possible rotational torque constriction.

Problem (Step 23): The clog is within the swivel.

Solution: Remove the microdialysis probe lead and run $\mathrm{ddH}_{2} \mathrm{O}$ through the swivel at $20 \mu \mathrm{L} / \mathrm{min}$. If perfusate is welling from the swivel, wait $5 \mathrm{~min}$ at $8 \mu \mathrm{L} / \mathrm{min}$. This will flush any particulate matter and/or bubbles in the swivel. Replace the swivel.

Problem (Step 23): The clog is within a section of FEP tubing.

Solution: Run at $20 \mu \mathrm{L} / \mathrm{min}$ through the isolated section of FEP for 5-10 min. Switch out the section of FEP tubing.

\section{DISCUSSION}

The in vivo microdialysis procedure described here has been used successfully to detect fluctuating neurosteroids in the auditory forebrain (Remage-Healey et al. 2008, 2012; Ikeda et al. 2012) and in the hippocampus (Rensel et al. 2012; Rensel and Schlinger 2013) of behaving adult zebra finches. Similar methods have been developed to examine changing levels of catecholamines in behaving zebra finches (e.g., Sasaki et al. 2006).

This protocol can be adapted to reverse-microdialyze (retrodialyze) drugs and steroidogenic compounds (Remage-Healey et al. 2008). When selecting candidate retrodialysis compounds, it is important to consider molecular mass. Proteins and other large molecules $>6 \mathrm{kDa}$ are likely to be bound up in the microdialysis tubing, but see Ulrich et al. (2013) for further discussion. Typical retrodialysis experiments involve collecting several serial baseline samples with aCSF perfusion, switching to a new syringe prefilled with drug dissolved in aCSF for 30-60 min, and then a period of washout in aCSF. When retrodialysis experiments are performed inside a sound-attenuation chamber, syringe changes can be conducted outside the chamber, allowing experimental manipulation without disturbing the animal. When switching to a new solution allow 1-2 min of high rate of 
M. Ikeda et al.

flow $(8-10 \mu \mathrm{L} / \mathrm{min})$ to confirm the perfusate is flowing and to eliminate air bubbles in the FEP tubing. Once flow is re-established the experiment can resume at the desired flow rate (i.e., $2 \mu \mathrm{L} / \mathrm{min}$ ). Automated liquid switches (obtained from CMA Microdialysis) can also be used. It is important to account for the "dead volume" of the microdialysis tubing and swivel, given the dimensions of FEP tubing and the total volume of perfusate passing through the swivel. Calculations of dead volume, factoring in the flow rate, allow for precise timing of sample collection during retrodialysis intervals.

The in vivo microdialysis methods described here can be used to study steroid signaling in the brain for a variety of experimental purposes. Thus, the combined study of neurochemistry and behavior in a vocal learning species now has a new set of powerful tools.

\section{RECIPE}

Artificial Cerebrospinal Fluid (aCSF) for Zebra Finch Brain

\begin{tabular}{lc} 
Reagent & Final concentration \\
\hline $\mathrm{NaCl}$ & $199 \mathrm{mM}$ \\
$\mathrm{NaHCO}$ & $26.2 \mathrm{mM}$ \\
$\mathrm{KCl}$ & $2.5 \mathrm{~mm}$ \\
$\mathrm{MgSO}_{4}$ & $1.0 \mathrm{mM}$ \\
$\mathrm{CaCl}_{2}$ & $2.5 \mathrm{mM}$ \\
$\mathrm{Glucose}$ & $11.0 \mathrm{mM}$ \\
Dissolve the reagents in ultrapure ddH & $\mathrm{O}(\sim 18 \mathrm{M} \Omega)$ and adjust the pH to $7.4-7.6$. Store \\
at $4^{\circ} \mathrm{C}$ in the dark for a maximum of 4 wk. Add $1 \%$ bovine serum albumin (BSA; Sigma- \\
Aldrich A1470) to 50-mL aliquots and filter (with 0.2- $\mu$ m syringe filters) to eliminate \\
suspended particles and reduce clogging of FEP tubing and probes.
\end{tabular}

\section{ACKNOWLEDGMENTS}

The authors would like to thank anonymous reviewers for reading over an earlier version of the manuscript, Nigel Maidment and Hoa Lam for initial training and expertise in adapting rodent microdialysis for birds, and Randi Oyama, Andrew Chao, Stephanie Dong, Ashley Paon, Ify Arinze, and Tom L. Cao for technical assistance. Support comes from NINDS K99/R00NS066179, NIMH061994, and the University of Massachusetts.

\section{REFERENCES}

Grandin T, ed. 2007. Livestock handling and transport, 3rd ed. CABI Publishing, Wallingford, Oxfordshire UK.

Ikeda M, McClure JR, Remage-Healey L. 2012. Catecholaminergic regulation of estradiol concentrations in the zebra finch auditory forebrain. Society for Neuroscience 2012, 92.07/SS16 (Abstr).

Remage-Healey L, Maidment NT, Schlinger BA. 2008. Forebrain steroid levels fluctuate rapidly during social interactions. Nat Neurosci 11: $1327-1334$

Remage-Healey L, Coleman MJ, Oyama RK, Schlinger BA. 2010. Brain estrogens rapidly strengthen auditory encoding and guide song preference in a songbird. Proc Natl Acad Sci 107: 3852-3857.

Remage-Healey L, Dong SM, Chao A, Schlinger BA. 2012. Sex-specific, rapid neuroestrogen fluctuations and neurophysiological actions in the songbird auditory forebrain. J Neurophysiol 107: 1621-1631.
Rensel MA, Salwiczek L, Hsaio CF, Xia S, Remage-Healey L, Schlinger BA. 2012. In vivo microdialysis reveals dynamics of estradiol production in the avian hippocampus. Society for Integrative and Comparative Biology 2012 Annual Meeting, Charleston, SC.

Rensel MA, Schlinger BA. 2013. Real-time measurement of hippocampal corticosterone in a songbird. Society for Integrative and Comparative Biology 2013 Annual Meeting, San Francisco, CA.

Sasaki A, Sotnikova TD, Gainetdinov RR, Jarvis ED. 2006. Social contextdependent singing-regulated dopamine. J Neurosci 26: 9010-9014.

Ulrich JD, Burchett JM, Restivo JL, Schuler DR, Verghese PB, Mahan TE, Landreth GE, Castellano JM, Jiang H, Cirrito JR, et al. 2013. In vivo measurement of apolipoprotein $\mathrm{E}$ from the brain interstitial fluid using microdialysis. Mol Neurodegener 8: doi: 10.1186/17501326-8-13. 


\section{In Vivo Detection of Fluctuating Brain Steroid Levels in Zebra Finches}

Maaya Ikeda, Michelle A. Rensel, Barney A. Schlinger and Luke Remage-Healey

Cold Spring Harb Protoc; doi: 10.1101/pdb.prot084616 originally published online October 23, 2014

\begin{tabular}{|c|c|}
\hline $\begin{array}{r}\text { Email Alerting } \\
\text { Service }\end{array}$ & Receive free email alerts when new articles cite this article - click here. \\
\hline $\begin{array}{l}\text { Subject } \\
\text { Categories }\end{array}$ & $\begin{array}{l}\text { Browse articles on similar topics from Cold Spring Harbor Protocols. } \\
\text { Behavioral Assays (99 articles) } \\
\text { Emerging Model Organisms (321 articles) } \\
\text { Immunodetection (34 articles) } \\
\text { Neuroscience, general (357 articles) }\end{array}$ \\
\hline
\end{tabular}

